\title{
ROLE OF OXIDATIVE DAMAGE AND MYOCARDIAL CELL INJURIES IN HUMAN SEVERE SEPSIS/SEPTIC SHOCK AFTER SURGICAL CORRECTION OF CONGENITAL HEART DISEASE IN INFANTS
}

\author{
M.S. Oliveira ${ }^{1}$, E.Z. Martinez ${ }^{1}$, L.C. Peres ${ }^{2}$, W.V.A. Vicente ${ }^{1}$, E.M. Floriano ${ }^{1}$, M.A. Rossi ${ }^{1}$, S.G. Ramos ${ }^{1}$ \\ ${ }^{1}$ University of Sao Paulo, Ribeirão Preto, Brazil, ${ }^{2}$ Sheffield Teaching Hospitals NHS Foundation Trust, \\ Sheffield, UK
}

Background and aims: Perioperative myocardial damage still remains the most common cause of morbidity and death after technically successful surgical correction. However, the situation is further complicated in patients with complex congenital cardiac malformations repair which developed septic shock/sepsis. The aim of the present study was to analyze the correlation between cell damage and oxidative stress in congenital heart disease (CHD) and septic patients' submitted to cardiac surgery.

Methods: The infants were divided into three groups: Group 1, 16 cases who underwent operations for CHD on cardiopulmonary bypass (CPB) with clinically diagnosed cardiogenic shock; Group 2, six cases who underwent operations for CHD on CPB in whom septic shock /sepsis developed, and Group 3 (control group), five cases with no CHD or sepsis/septic shock who died from other causes. The myocardial injuries and oxidative stress mechanisms were assessed by histopathology and immunohistochemistry for hydroxinonenal (4-HNE) and nitrotyrosine (3-NT), and were quantified by morphometrical analyses.

Results/conclusions: Contraction band necrosis and dystrophic calcification were found primarily in infants of Group 1. Colliquative myocytolysis, coagulation necrosis and healing were prominent in Group 2. The control group showed no significant myocardial lesions. Lipid peroxidation demonstrated by 4-HNE was the principal mechanism of oxidative stress accounting for the myocardial lesions observed in group 1 . On the other hand, an increased concentration of 3-NT proteins adducts were observed in human septic hearts, suggesting that protein nitration could be the most prevalent oxidative stress mechanism found in septic patients. 